\title{
Divergences entre les données administratives sur les infections associées aux soins de santé et les données issues de la surveillance active au Canada
}

\author{
Virginie Boulanger ${ }^{1,2}$, Étienne Poirier ${ }^{1,2}$, Anne MacLaurin ${ }^{3}$, Caroline Quach ${ }^{1,2,4,5 *}$
}

\section{Résumé}

Contexte : Bien que le Canada dispose à la fois d'un système national de surveillance active et de données administratives pour la surveillance passive des infections associées aux soins de santé (IASS), chacun des deux systèmes présente des forces et des faiblesses au niveau de la collecte et de la communication des données. La surveillance active et la surveillance passive fonctionnent de manière indépendante, ce qui donne parfois des résultats divergents. Pour comprendre les divergences qui existent entre les données administratives sur la santé et les données issues de la surveillance active, un examen de la portée a été réalisé.

Méthodes : Des recherches ont été menées dans les bases de données Medline, Embase et Cumulative Index to Nursing and Allied Health Literature, ainsi que dans la littérature grise pour trouver des études en anglais et en français évaluant l'utilisation de données administratives, seules ou en comparaison avec les données issues de la surveillance traditionnelle et réalisées au Canada entre 1995 et le 2 novembre 2020. Après avoir extrait les renseignements pertinents des articles sélectionnés, un résumé descriptif des résultats a été présenté, accompagné de suggestions pour améliorer les systèmes de surveillance, dans le but d'optimiser la qualité globale des données.

Résultats : Seize articles répondaient aux critères d'inclusion, dont douze études observationnelles et quatre revues systématiques. Ces études ont démontré que l'utilisation d'une seule source de données administratives n'était pas assez précise pour la surveillance des IASS, par rapport à la surveillance active traditionnelle. Cependant, la combinaison de différentes sources de données ou la combinaison de données administratives et de données issues de la surveillance active en améliore la précision. Les systèmes de surveillance électronique peuvent également renforcer la surveillance en augmentant la capacité à détecter les IASS potentielles.

Conclusion : Bien que la surveillance active des IASS ait produit les résultats les plus précis et demeure la norme d'excellence en la matière, l'intégration des données issues de la surveillance active et passive peut être optimisée. Les données administratives peuvent être utilisées pour renforcer la surveillance active traditionnelle. De futures études sont nécessaires pour évaluer la faisabilité et les avantages des solutions potentielles proposées pour l'utilisation des données administratives pour la surveillance et la déclaration des IASS au Canada.
Cette oeuvre est mise à la disposition selon les termes de la licence internationale Creative Commons Attribution 4.0

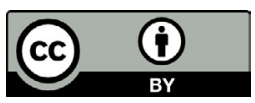

Affiliations

1 Département de microbiologie, infectiologie et immunologie, Faculté de médecine, Université de Montréal, Montréal, QC

${ }^{2}$ Centre de recherche du $\mathrm{CHU}$ Sainte-Justine, Montréal, QC

${ }^{3}$ Excellence en santé Canada

${ }^{4}$ Département de médecine de laboratoire pédiatrique, $\mathrm{CHU}$ Sainte-Justine, Montréal, QC

${ }^{5}$ Prévention et contrôle des infections, CHU Sainte-Justine, Montréal, QC

*Correspondance :

c.quach@umontreal.ca

Citation proposée : Boulanger V, Poirier E, MacLaurin A, Quach C. Divergences entre les données

administratives sur les infections associées aux soins de santé et les données issues de la surveillance active au

Canada. Relevé des maladies transmissibles au Canada 2022;48(1):4-18.

https://doi.org/10.14745/ccdr.v48i01a02f

Mots-clés : surveillance, infection associée aux soins de santé, données administratives 


\section{Introduction}

Chaque année, de nombreux Canadiens contractent une infection au cours d'un séjour à l'hôpital, ce qui augmente les taux de morbidité et de mortalité, en plus de représenter un coût financier pour le système de santé (1). Ces IASS sont évitables, mesurables et constituent l'événement indésirable le plus fréquemment signalé dans le domaine des soins de santé à l'échelle internationale. Chaque année, on estime que 220000 patients canadiens développent une IASS (2). De nombreuses IASS sont désormais causées par des organismes résistants aux antimicrobiens, ce qui les rend difficiles à traiter. L'Agence de la santé publique du Canada (l'Agence) estime qu'environ $2 \%$ des patients admis dans les grands hôpitaux universitaires canadiens contracteront une infection par un organismes résistants aux antimicrobiens pendant leur séjour à I'hôpital (3). La surveillance, qui inclut le suivi et le signalement des IASS, est une composante essentielle de la prévention et du contrôle des infections, et elle doit être renforcée à l'échelle nationale. Bien que la maladie à coronavirus 2019 (COVID-19) ne soit pas à l'origine une IASS, la pandémie actuelle a révélé à quel point il est crucial de disposer de données fiables et cohérentes afin de répondre adéquatement à l'infection. Lorsqu'on lui a demandé de fournir des projections concernant l'évolution du virus de la COVID-19, le premier ministre Trudeau a déclaré que [traduction] « ...l'incohérence des données provenant de l'ensemble du Canada explique en partie le retard accusé pour offrir une vue d'ensemble de la situation au pays » (4).

Au Canada, l'Agence recueille des données nationales sur de multiples IASS par l'intermédiaire du Programme canadien de surveillance des infections nosocomiales (PCSIN), un programme établi en 1994 sous forme de partenariat entre l'Agence, l'Association pour la microbiologie médicale et l'infectiologie du Canada et certains hôpitaux sentinelles de partout au Canada (5). Les objectifs du PCSIN sont de fournir des points de référence à l'échelle nationale et régionale, d'identifier les tendances pour certaines IASS et certains organismes résistants aux antimicrobiens, ainsi que de fournir des informations clés pour l'élaboration de programmes et de politiques de prévention et de contrôle des infections, tant au niveau fédéral, provincial et territorial (5). Actuellement, le réseau du PCSIN comprend 87 hôpitaux sentinelles de soins de courte durée, situés dans dix provinces et un territoire. L'objectif du réseau est de faire en sorte que tous les hôpitaux canadiens de soins de courte durée adoptent les définitions des IASS formulées à des fins de surveillance par le PCSIN et contribuent à fournir des données au système de surveillance national (2). Malgré l'ambition existante d'étendre le programme de surveillance, le PCSIN est limité par 1) la capacité de financement, 2) le manque de ressources humaines disponibles pour participer à la surveillance nationale (2) et 3) parce que la plupart des hôpitaux font déjà rapport auprès de leur gouvernement provincial et ne tiennent pas à soumettre les données deux fois. Par conséquent, les taux d'IASS présentés dans le cadre du PCSIN peuvent ne pas donner une image juste et complète de la situation; de plus, certains segments de la population hospitalière canadienne sont sous-représentés, par exemple les petits hôpitaux communautaires (6).

Les statistiques nationales de l'Agence sur les IASS ne comprennent que les données des hôpitaux qui participent au PCSIN, car ils suivent tous des définitions de cas, des méthodes et des rapports de cas normalisés. À l'heure actuelle, les taux d'IASS déclarés par les provinces et les territoires ou affichés par les hôpitaux individuels ne peuvent être combinés, car les définitions de cas, les méthodes de collecte de données et le calcul des taux varient d'un hôpital à l'autre et entre les provinces et les territoires (2). La surveillance active est effectuée par les praticiens de la prévention et du contrôle des infections $(\mathrm{PCl})$ et chaque province, territoire, région administrative ou hôpital peut établir ses propres protocoles de surveillance en fonction de l'épidémiologie et des ressources locales, ce qui rend difficile l'évaluation des efforts déployés pour l'amélioration du système et la comparaison des taux d'IASS dans les différents hôpitaux canadiens (7).

D'autre part, le Canada dispose d'une multitude de données administratives sur la santé, notamment les registres d'assurance, les soins donnés aux patients hospitaliers, les statistiques de l'état civil, les ordonnances de médicaments et les systèmes de dossiers médicaux électroniques (8). L'exploration du potentiel d'intégration de ces divers ensembles de données administratives sur la santé pourrait fournir un portrait plus fidèle des IASS au Canada.

La base de données sur les congés des patients (BDCP), hébergée à I'Institut canadien d'information sur la santé (ICIS), rassemble des renseignements démographiques et cliniques provenant des sommaires d'hospitalisation des patients de tous les établissements de soins de courte durée au Canada, sauf ceux du Québec (le Québec a sa propre base de données sur les congés des patients - Maintenance et exploitation des données pour l'étude de la clientèle hospitalière (MED-ÉCHO) - qui relève de la base de données sur la morbidité hospitalière de I'ICIS) (9). Les informations de chacun des hôpitaux sont saisies dans la base de données par des professionnels du codage et sont utilisées par I'ICIS pour produire des données et des rapports analytiques. Le programme de qualité des données et de l'information de l'ICIS est reconnu à l'échelle internationale pour son exhaustivité et ses normes élevées (10). Cependant, les sommaires d'hospitalisation ne sont pas standardisés à l'échelle nationale et reflètent seulement ce qui est inscrit dans le sommaire par le médecin traitant. L'ICIS pourrait toutefois devenir un partenaire potentiel pour soutenir la collecte de données et la déclaration des IASS dans les hôpitaux offrant des soins de courte durée. Nous avons réalisé un examen de la portée dans le but d'identifier les lacunes existantes entre les données administratives et les données issues de la surveillance active pour les IASS, ainsi que pour proposer des stratégies 
d'intégration qui pourraient possiblement permettre d'optimiser les données.

\section{Méthodes}

\section{Question de recherche}

La question au centre de notre recherche était la suivante:

"Quelles sont les divergences entre les données administratives sur les IASS et les données issues de la surveillance active au Canada? ». Les sous-questions qui se posaient dans le cadre de notre recherche étaient : Les données administratives sont-elles valables pour la surveillance des IASS? Pour chaque type d'IASS, quelles sont les divergences entre les données administratives et les données de surveillance des hôpitaux? Nous avons réalisé cet examen de la portée en nous conformant aux lignes directrices de l'extension PRISMA pour les examens de la portée (PRISMA ScR) (11).

Aux fins de cet examen de la portée, les IASS comprennent les infections à Clostridioides difficile ( $C$. difficile; ICD), les infections du système sanguin associées aux cathéters centraux, les infections des voies urinaires associées aux cathéters ou les infections des voies urinaires, les Staphylococcus aureus résistants à la méthicilline (SARM), les entérocoques résistants à la vancomycine, les entérobactériacées résistantes aux carbapénèmes, les organismes résistants aux antimicrobiens, les infections du sang, les infections du site opératoire (ISO) et les pneumonies associées à la ventilation assistée.

\section{Littérature pertinente}

Nous avons effectué une recherche qui a été développée en collaboration avec une bibliothécaire spécialisée en recherche médicale. Les critères d'inclusion visaient les articles évaluant la surveillance passive des IASS (variées ou spécifiques) au Canada. Nous avons inclus à notre recherche les articles (études qualitatives, quantitatives et à méthodes mixtes) ayant été publiés au Canada entre 1995 et le 2 novembre 2020.

La stratégie de recherche incluait des termes relatifs au lieu (Canada), à la surveillance, à la source des données et aux IASS. En outre, nous avons effectué une deuxième recherche avec les mêmes termes (à l'exception des termes relatifs au lieu), en incluant uniquement les revues systématiques.

Un premier processus de sélection a été effectué pour identifier les bases de données contenant des études pertinentes; trois bases de données électroniques ont été consultées : MEDLINE, EMBASE et Cumulative Index to Nursing and Allied Health Literature. Elles ont été consultées en anglais et en français, sans restriction de date. Les stratégies de recherche ont été créées sur MEDLINE, puis adaptées pour les autres bases de données (données supplémentaires S1). Après avoir éliminé les doublons, deux examinateurs ont indépendamment examiné les articles de référence pour les filtrer en fonction du titre et du résumé. Les articles sélectionnés ont ensuite été étudiés pour leur admissibilité au niveau du texte intégral. Le premier examinateur a également effectué une recherche manuelle dans la littérature grise et a dépouillé la liste de référence de toutes les études identifiées, de façon à cibler tous les articles qui n'avaient pas été initialement repérés par la recherche électronique. Les différends entre examinateurs ont été résolus par la discussion jusqu'à ce qu'un consensus soit atteint.

\section{Extraction des données et évaluation de la qualité}

Un fichier de données électronique a été développé avec DistillerSR (Evidence Partners, Ottawa, Canada) pour cet examen de la portée. Les données suivantes ont été extraites de chaque article : renseignements généraux, détails de l'étude, types d'IASS et type de surveillance, source des données, résultats.

Les deux examinateurs ont évalué le risque de biais pour chacune des études ainsi que leur qualité, en utilisant l'outil ROBINS-I pour les études non randomisées (12), et l'outil AMSTAR-2 pour les revues systématiques (13). Dans l'ensemble, les études ont été classées comme présentant un risque de biais faible, modéré ou élevé. Tout désaccord ou toute contradiction entre les examinateurs a été résolu par la discussion. La collecte complète des données et les éléments d'évaluation de la qualité sont présentés dans les données supplémentaires S2.

\section{Analyse des données}

Une approche qualitative descriptive a été choisie pour synthétiser les données recueillies. Les caractéristiques des principales études, le sommaire des statistiques de performance et les résultats d'évaluation de la qualité ont été résumés dans des tableaux. Nous avons présenté un sommaire des résultats pour chaque étude, en les regroupant par catégorie selon le type de données administratives utilisées et la portée de l'étude. Nous nous sommes concentrés sur la manière dont les données administratives ont été utilisées pour la surveillance des IASS, sur la divergence des résultats avec ceux issus de la surveillance traditionnelle et sur le fait que les auteurs recommandent ou non d'utiliser les données administratives pour optimiser la surveillance. Une synthèse des revues systématiques a également été présentée, les études ayant été classées comme évaluant la validité des données administratives, ou évaluant la validité des systèmes de surveillance électroniques.

\section{Résultats}

Au total, 1316 études ont été ciblées par la recherche électronique, et 12 lors de la recherche manuelle. Après avoir éliminé les doublons, 1102 études restaient, dont 104 ont été sélectionnées pour un examen du texte intégral. Finalement, 16 études identifiées à partir de la recherche électronique ont 
été incluses dans l'examen de la portée; douze étant des études observationnelles (14-25), et quatre des revues systématiques (26-29) (figure 1).

\section{Caractéristiques de l'étude}

Sur les 12 études observationnelles incluses, six portaient sur les ISO, trois sur les ICD, deux sur le SARM et une sur les infections du sang. Ces études ont toutes été réalisées entre 2009 et 2020, et huit d'entre elles proviennent de l'Alberta. Les données administratives étaient comparées avec les données de surveillance des hôpitaux dans sept études, et le couplage des données était utilisé pour sept autres. La BDCP a été utilisée comme source de données administratives (seule ou combinée à d'autres sources) dans toutes les études. Les principales caractéristiques de toutes les études incluses sont résumées dans le tableau 1.

Quatre revues systématiques ont également été incluses : trois sur l'utilisation du système de surveillance électronique (SSE), et une sur l'utilisation des données administratives pour la
Figure 1 : Diagramme illustrant la sélection des études

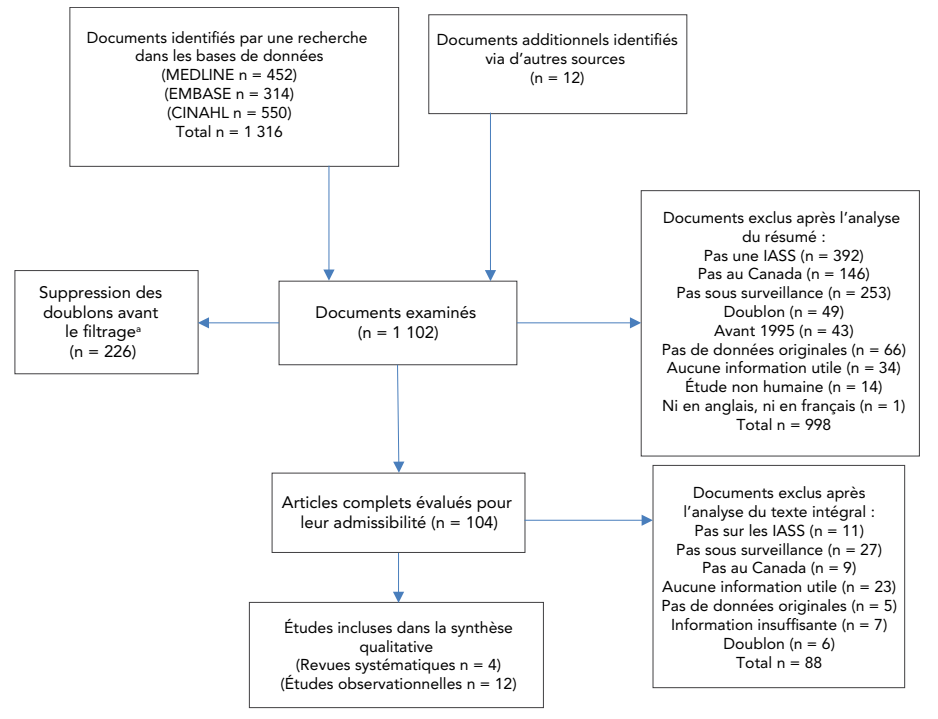

Abréviations : CINAHL, Cumulative Index to Nursing and Allied Health Literature; IASS, infections associées aux soins de santé

a Utilisation du logiciel EndNote X9.1.1

\section{Tableau 1 : Études observationnelles-Caractéristiques de l'étude}

\begin{tabular}{|c|c|c|c|c|c|c|}
\hline $\begin{array}{l}\text { Auteur } \\
\text { principal, } \\
\text { année }\end{array}$ & $\begin{array}{l}\text { Conception } \\
\text { de l'étude }\end{array}$ & $\begin{array}{l}\text { Population étudiée et taille de } \\
\text { l'échantillon }(n=)\end{array}$ & $\begin{array}{c}\text { Source de } \\
\text { données } \\
\text { administratives }\end{array}$ & Condition(s) & Province(s) & $\begin{array}{l}\text { Risque } \\
\text { de biais }\end{array}$ \\
\hline $\begin{array}{l}\text { Crocker, } \\
2020\end{array}$ & $\begin{array}{l}\text { Étude de } \\
\text { cohorte }\end{array}$ & $\begin{array}{l}\text { Tous les cas index de laminectomie et de } \\
\text { fusion vertébrale en Alberta, de } 2008 \text { à } \\
2015(n=21222)\end{array}$ & $\mathrm{BDCP}+\mathrm{SNISA}^{\mathrm{a}}$ & $\begin{array}{l}\text { Infection du site } \\
\text { opératoire }\end{array}$ & Alberta & Faible \\
\hline $\begin{array}{l}\text { Ramirez- } \\
\text { Mendoza, } \\
2016\end{array}$ & $\begin{array}{l}\text { Étude de } \\
\text { cohorte }\end{array}$ & $\begin{array}{l}\text { Tous les patients en soins de courte durée } \\
\text { en Alberta et en Ontario, d'avril } 2012 \text { à } \\
\text { mars } 2013(n=217)^{b}\end{array}$ & $\mathrm{BDCP}$ & $\begin{array}{l}\text { Staphylococcus aureus } \\
\text { résistant à la méthicilline }\end{array}$ & $\begin{array}{l}\text { Alberta et } \\
\text { Ontario }\end{array}$ & Faible \\
\hline $\begin{array}{l}\text { Pfister, } \\
2020\end{array}$ & $\begin{array}{l}\text { Étude de } \\
\text { cohorte }\end{array}$ & 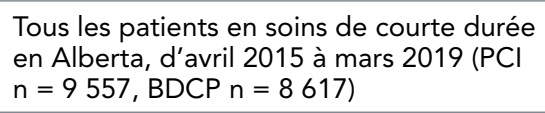 & $\mathrm{BDCP}$ & Clostridioides difficile & Alberta & Faible \\
\hline $\begin{array}{l}\text { Rennert- } \\
\text { May, } 2018\end{array}$ & $\begin{array}{l}\text { Étude de } \\
\text { cohorte }\end{array}$ & $\begin{array}{l}\text { Tous les cas d'arthroplastie primaire de la } \\
\text { hanche ou du genou en Alberta, d'avril } \\
2012 \text { à mars } 2015 \text { ( } n=24512)\end{array}$ & $\mathrm{BDCP}$ & $\begin{array}{l}\text { Infection du site } \\
\text { opératoire }\end{array}$ & Alberta & Faible \\
\hline $\begin{array}{l}\text { Almond, } \\
2019\end{array}$ & $\begin{array}{l}\text { Étude de } \\
\text { cohorte }\end{array}$ & $\begin{array}{l}\text { Tous les patients en soins de courte durée } \\
\text { en Alberta, d'avril } 2015 \text { à mars } 2017 \\
(\mathrm{n}=4737)\end{array}$ & $\begin{array}{l}\mathrm{BDCP}+\text { données } \\
\text { de laboratoire }\end{array}$ & Clostridioides difficile & Alberta & Faible \\
\hline Rusk, 2016 & $\begin{array}{l}\text { Étude de } \\
\text { cohorte }\end{array}$ & $\begin{array}{l}\text { Tous les cas d'arthroplastie primaire de la } \\
\text { hanche ou du genou en Alberta, d'avril } \\
2013 \text { à juin } 2014(\mathrm{n}=11774)\end{array}$ & $\mathrm{BDCP}+\mathrm{SNISA}^{\mathrm{a}}$ & $\begin{array}{l}\text { Infection du site } \\
\text { opératoire }\end{array}$ & Alberta & Faible \\
\hline $\begin{array}{l}\text { Daneman, } \\
2011\end{array}$ & $\begin{array}{l}\text { Étude de } \\
\text { cohorte }\end{array}$ & $\begin{array}{l}\text { Tous les cas d'accouchement par } \\
\text { césarienne au Sunnybrook Health Science } \\
\text { Centre, de janvier } 2008 \text { à décembre } 2009 \\
(n=2532)\end{array}$ & $\begin{array}{l}\text { BDCP + SNISA + } \\
\text { déclarations des } \\
\text { médecins }^{a}\end{array}$ & $\begin{array}{l}\text { Infection du site } \\
\text { opératoire }\end{array}$ & Ontario & Faible \\
\hline $\begin{array}{l}\text { Lethbridge, } \\
2019\end{array}$ & $\begin{array}{l}\text { Étude de } \\
\text { cohorte }\end{array}$ & $\begin{array}{l}\text { Tous les cas de chirurgie de } \\
\text { remplacement de la hanche ou du genou } \\
\text { en Nouvelle-Écosse, de } 2001 \text { à } 2015 \\
(\mathrm{n}=36 \text { 140) }\end{array}$ & $\begin{array}{l}\text { BDCP + SNISA + } \\
\text { déclarations des } \\
\text { médecins }\end{array}$ & $\begin{array}{l}\text { Infection du site } \\
\text { opératoire }\end{array}$ & $\begin{array}{l}\text { Nouvelle- } \\
\text { Écosse }\end{array}$ & Faible \\
\hline Leal, 2010 & $\begin{array}{l}\text { Étude de } \\
\text { cohorte }\end{array}$ & $\begin{array}{l}\text { Tous les patients adultes de la région } \\
\text { sanitaire de Calgary en } 2005 \text { (échantillon } \\
\text { de } n=2281 \text { ) }\end{array}$ & $\begin{array}{l}\text { Laboratoire } \\
\text { PathNet, de } \\
\text { Cerner }+ \text { Oracle }^{c}\end{array}$ & Infection du sang & Alberta & Faible \\
\hline Lee, 2019 & $\begin{array}{l}\text { Étude de } \\
\text { cohorte }\end{array}$ & $\begin{array}{l}\text { Tous les patients adultes de quatre } \\
\text { établissements de soins de courte durée } \\
\text { pour adultes de la région de Calgary, } \\
\text { d'avril } 2011 \text { à mars } 2017 \text { ( } n=2430)\end{array}$ & BDCP & $\begin{array}{l}\text { Staphylococcus aureus } \\
\text { résistant à la méthicilline }\end{array}$ & Alberta & Faible \\
\hline
\end{tabular}


Tableau 1 : Études observationnelles-Caractéristiques de l'étude (suite)

\begin{tabular}{|c|c|c|c|c|c|c|}
\hline $\begin{array}{l}\text { Auteur } \\
\text { principal, } \\
\text { année }\end{array}$ & $\begin{array}{l}\text { Conception } \\
\text { de l'étude }\end{array}$ & $\begin{array}{l}\text { Population étudiée et taille de } \\
\text { l'échantillon }(n=)\end{array}$ & $\begin{array}{c}\text { Source de } \\
\text { données } \\
\text { administratives }\end{array}$ & Condition(s) & Province(s) & $\begin{array}{l}\text { Risque } \\
\text { de biais }\end{array}$ \\
\hline $\begin{array}{l}\text { Daneman, } \\
2009\end{array}$ & $\begin{array}{l}\text { Étude de } \\
\text { cohorte }\end{array}$ & $\begin{array}{l}\text { Tous les patients âgés hospitalisés pour } \\
\text { une chirurgie non urgente en Ontario, } \\
\text { d'avril } 1992 \text { à mars } 2006 \text { ( } n=469349 \text { ) }\end{array}$ & $\begin{array}{l}\text { BDCP + Régime } \\
\text { d'assurance- } \\
\text { maladie de } \\
\text { l'Ontario + Base } \\
\text { de données du } \\
\text { Programme de } \\
\text { médicaments de } \\
\text { l'Ontario }\end{array}$ & $\begin{array}{l}\text { Infection du site } \\
\text { opératoire }\end{array}$ & Ontario & Faible \\
\hline $\begin{array}{l}\text { Daneman, } \\
2012\end{array}$ & $\begin{array}{l}\text { Étude de } \\
\text { cohorte }\end{array}$ & $\begin{array}{l}\text { Tous les patients (âgés de plus d'un an) } \\
\text { admis dans un hôpital de soins de courte } \\
\text { durée en Ontario entre avril } 2002 \text { et mars } \\
2010 \text { ( } n=180)^{b}\end{array}$ & BDCPa & Clostridioides difficile & Ontario & Faible \\
\hline
\end{tabular}

Abréviations : BDCP, base de données sur les congés des patients; $\mathrm{PCl}$, prévention et contrôle des infections; SNISA, Système national d'information sur les soins ambulatoires

a Par rapport aux données issues de la surveillance active

b Nombre d'hôpitaux

Système de base de données Oracle du dépôt régional

surveillance des IASS. Toutes les revues comprenaient au moins un article provenant du Canada. Les caractéristiques de chaque étude sont résumées dans le tableau 2.

\section{Risque de biais au sein de l'étude}

Le risque de biais dans les études observationnelles a été évalué à l'aide de l'outil ROBIN-1 (tableau 1). La plupart de ces études utilisaient une méthodologie similaire, mais ne fournissaient pas d'informations sur les données manquantes (tableau supplémentaire S3). Cependant, toutes ces études ont été évaluées comme présentant un faible risque de biais.

Les revues systématiques ont été évaluées à l'aide de l'outil AMSTAR-2 (tableau 2, tableau supplémentaire S4). Un seul article a été considéré comme présentant un risque modéré de biais, car il ne faisait pas état du protocole utilisé et ne décrivait pas les études incluses de manière suffisamment détaillée. Trois articles ont été considérés comme présentant un risque élevé

\section{Tableau 2 : Revue systématique-Caractéristiques des études}

\begin{tabular}{|c|c|c|c|c|c|c|}
\hline $\begin{array}{l}\text { Auteur } \\
\text { principal, } \\
\text { année }\end{array}$ & $\begin{array}{l}\text { Nombre } \\
\text { d'études } \\
\text { incluses, } \\
\text { année }\end{array}$ & Objectif & Bases de données & Conclusion & $\begin{array}{c}\text { Autres } \\
\text { renseignements }\end{array}$ & $\begin{array}{l}\text { Risque de } \\
\text { biais }\end{array}$ \\
\hline $\begin{array}{l}\text { Van Mourik, } \\
2015\end{array}$ & $\begin{array}{l}57 \text { études, de } \\
1995 \text { à } 2013\end{array}$ & $\begin{array}{l}\text { Exactitude } \\
\text { des données } \\
\text { administratives } \\
\text { utilisées pour la } \\
\text { surveillance des IASS }\end{array}$ & $\begin{array}{l}\text { Medline, Embase, } \\
\text { CINAHL, Cochrane }\end{array}$ & $\begin{array}{l}\text { Les données } \\
\text { administratives } \\
\text { avaient une } \\
\text { précision limitée et } \\
\text { très variable }\end{array}$ & $\begin{array}{l}1 \text { étude sur } 3 \text { parmi } \\
\text { celles incluses } \\
\text { présentaient des limites } \\
\text { méthodologiques } \\
\text { importantes }\end{array}$ & Modéré \\
\hline Leal, 2008 & $\begin{array}{l}24 \text { études, de } \\
1980 \text { à } 2007\end{array}$ & $\begin{array}{l}\text { Identifier et examiner } \\
\text { la littérature } \\
\text { publiée visant à } \\
\text { évaluer la validité } \\
\text { du SSE par rapport } \\
\text { à la surveillance } \\
\text { conventionnelle }\end{array}$ & Medline & $\begin{array}{l}\text { La surveillance } \\
\text { électronique a } \\
\text { une utilité notable } \\
\text { par rapport à } \\
\text { la surveillance } \\
\text { conventionnelle }\end{array}$ & $\begin{array}{l}\text { Aucune évaluation de } \\
\text { la qualité des études } \\
\text { incluses }\end{array}$ & Élevé \\
\hline Freeman, 2013 & $\begin{array}{l}24 \text { études, de } \\
2000 \text { à } 2011\end{array}$ & $\begin{array}{l}\text { Évaluer l'utilité du SSE } \\
\text { pour la surveillance et } \\
\text { la détection des IASS }\end{array}$ & $\begin{array}{l}\text { Medline, Cochrane, } \\
\text { Ovid, Embase, Web of } \\
\text { science, Scopus, JSTOR, } \\
\text { Wiley Online Library, } \\
\text { BIOSIS Preview }\end{array}$ & $\begin{array}{l}\text { Les hôpitaux } \\
\text { doivent } \\
\text { développer et } \\
\text { utiliser des SSE } \\
\text { pour les IASS }\end{array}$ & $\begin{array}{l}\text { La majorité des } \\
\text { études insistent sur le } \\
\text { couplage des bases de } \\
\text { données électroniques }\end{array}$ & Élevé \\
\hline Streefkerk, 2020 & $\begin{array}{l}78 \text { études, } \\
\text { jusqu'en } \\
\text { janvier } 2018\end{array}$ & $\begin{array}{l}\text { Donner un aperçu } \\
\text { de l'état actuel des } \\
\text { SSE, en évaluant les } \\
\text { performances et la } \\
\text { qualité }\end{array}$ & $\begin{array}{l}\text { Embase, Medline, } \\
\text { Cochrane, Web of } \\
\text { Science, Scopus, } \\
\text { CINAHL, Google Scholar }\end{array}$ & $\begin{array}{l}\text { Avec une } \\
\text { sensibilité } \\
\text { généralement } \\
\text { élevée mais une } \\
\text { spécificité variable, } \\
\text { les SSE n'ont pas } \\
\text { encore atteint leur } \\
\text { plein potentiel } \\
\text { et doivent être } \\
\text { perfectionnés }\end{array}$ & $\begin{array}{l}\text { Les auteurs ont } \\
\text { sélectionné les } 10 \\
\text { meilleures études } \\
\text { pouvant constituer } \\
\text { une référence pour le } \\
\text { développement des } \\
\text { SSE }\end{array}$ & Élevé \\
\hline
\end{tabular}

Abréviations : CINAHL, Cumulative Index to Nursing and Allied Health Literature; IASS, infections associées aux soins de santé; SSE, système de surveillance électronique 
de biais, parce que certains ne faisaient pas état du protocole utilisé, ou de l'évaluation du risque de biais, de la qualité ou de I'hétérogénéité des études incluses.

\section{Résumé des conclusions}

\section{Études utilisant une base de données administrative en comparaison avec les données issues de la surveillance active}

Des études de validation ont montré que la BDCP utilisée seule pour collecter les cas d'IASS n'est pas valide par rapport à la surveillance active traditionnelle des hôpitaux par les équipes de
PCl. Par exemple, Rennert-May et al. (17) ont évalué la validité de l'utilisation de la base de données administrative des codes CIM-10 (BDCP) pour identifier les ISO complexes dans les trois mois suivant une arthroplastie de la hanche ou du genou. L'étude a révélé que les codes $\mathrm{CIM}$ de la BDCP étaient très spécifiques $(99,5 \%)$ mais avaient une sensibilité de $85,3 \%$ et une valeur prédictive positive de seulement $63,6 \%$. Ils ont conclu que la BDCP n'était pas en mesure de déterminer avec précision si une personne avait une ISO selon la définition donnée pour la surveillance (tableau 3). Pfister et al. (15) sont arrivés à la même conclusion avec une étude de validation sur la BDCP collectant les cas d'ICD. Le taux d'ICD de la BDCP était de $28 \%$ plus élevé que celui déterminé par la surveillance de $\mathrm{PCl}$, ce qui démontre que l'utilisation de la BDCP semble inadéquate pour obtenir le

Tableau 3 : Études observationnelles-Résumé des statistiques de performance

\begin{tabular}{|c|c|c|c|c|c|c|c|c|}
\hline \multirow[b]{2}{*}{$\begin{array}{l}\text { Auteur } \\
\text { principal, } \\
\text { année }\end{array}$} & \multirow[b]{2}{*}{ Comparateur } & \multicolumn{6}{|c|}{ Résultats } & \multirow[b]{2}{*}{ Conclusion } \\
\hline & & Taux d'infection & $\begin{array}{l}\text { VP, FP, FN, } \\
\text { VN, Total }\end{array}$ & Sensibilité & Spécificité & $\begin{array}{l}\text { Valeur } \\
\text { prédictive } \\
\text { positive }\end{array}$ & $\begin{array}{c}\text { Valeur } \\
\text { prédictive } \\
\text { négative }\end{array}$ & \\
\hline $\begin{array}{l}\text { Crocker, } \\
2020\end{array}$ & $\begin{array}{l}\text { BDCP + SNISA } \\
\text { comparées avec } \\
\text { les données } \\
\text { de surveillance } \\
\text { traditionnelles } \\
\text { publiées }\end{array}$ & $\begin{array}{l}2,7 \text { pour } 100 \\
\text { procédures de } \\
\text { laminectomie } \\
3,2 \text { pour } 100 \\
\text { procédures de } \\
\text { fusion vertébrale }\end{array}$ & s.o. & s.o. & s.o. & s.o. & s.o. & $\begin{array}{l}\text { Le taux rapporté par les } \\
\text { données administratives } \\
\text { était similaire au } \\
\text { taux publié apporté } \\
\text { par la surveillance } \\
\text { traditionnelle } \\
\text { Nécessité d'une étude } \\
\text { de validation pour } \\
\text { vérifier les résultats }\end{array}$ \\
\hline $\begin{array}{l}\text { Ramirez- } \\
\text { Mendoza, } \\
2016\end{array}$ & $\begin{array}{l}\text { Comparaison } \\
\text { entre la BDCP } \\
\text { et les données } \\
\text { de } \mathrm{PCl}\end{array}$ & $\begin{array}{l}\text { Alberta (cas pour } \\
10000 \text { jours- } \\
\text { patients) } \\
\mathrm{BDCP}: 0,43 \\
\mathrm{PCl}: 0,91 \\
\text { Ontario (cas pour } \\
10000 \text { jours- } \\
\text { patients) } \\
\mathrm{BDCP}: 0,25 \\
\mathrm{PCl}: 0,21\end{array}$ & s.o. & s.o. & s.o. & s.o. & s.o. & $\begin{array}{l}\text { L'utilisation de la } \\
\text { corrélation de Pearson } \\
\text { a permis de démontrer } \\
\text { la comparabilité des } \\
\text { données administratives } \\
\text { et des données de } \\
\text { surveillance de } \mathrm{PCl}\end{array}$ \\
\hline $\begin{array}{l}\text { Pfister, } \\
2020\end{array}$ & $\begin{array}{l}\text { Comparaison } \\
\text { entre la BDCP } \\
\text { et les données } \\
\text { de } \mathrm{PCl}\end{array}$ & $\begin{array}{l}\text { BDCP : 6,49 pour } \\
1000 \text { admissions } \\
\mathrm{PCl}: 5,06 \text { pour } \\
1000 \text { admissions }\end{array}$ & $\begin{array}{l}5477 \mathrm{VP} \\
1400 \mathrm{FP} \\
968 \mathrm{FN} \\
344 \mathrm{TN} \\
\text { Total } 8169\end{array}$ & $85 \%$ & s.o. & $80 \%$ & s.o. & $\begin{array}{l}\text { La BDCP était } \\
\text { modérément sensible, } \\
\text { mais probablement } \\
\text { inadéquate pour obtenir } \\
\text { l'incidence réelle }\end{array}$ \\
\hline $\begin{array}{l}\text { Rennert- } \\
\text { May, } 2018\end{array}$ & $\begin{array}{l}\text { Comparaison } \\
\text { entre la BDCP } \\
\text { et les données } \\
\text { de } \mathrm{PCl}\end{array}$ & s.o. & $\begin{array}{l}220 \mathrm{VP} \\
126 \mathrm{FP} \\
38 \mathrm{FN} \\
24128 \mathrm{VN} \\
\text { Total } \\
24512 \\
\end{array}$ & $85,3 \%$ & $99,5 \%$ & $63,6 \%$ & $99,8 \%$ & $\begin{array}{l}\text { Les données } \\
\text { administratives } \\
\text { présentaient des } \\
\text { résultats raisonnables, } \\
\text { mais la surveillance de } \\
\text { PCl était supérieure }\end{array}$ \\
\hline $\begin{array}{l}\text { Almond, } \\
2019\end{array}$ & $\begin{array}{l}\text { BDCP + données } \\
\text { de laboratoire } \\
\text { comparées avec } \\
\text { les données de } \\
\mathrm{PCl}\end{array}$ & $\begin{array}{l}\text { BDCP/lab (par } \\
10000 \\
\text { jours-patients) } \\
4,96 \text { pour les IASS } \\
\mathrm{PCI} \text { (pour } 10000 \\
\text { jours-patients) } \\
3,46 \text { pour les IASS }\end{array}$ & $\begin{array}{l}1998 \mathrm{VP} \\
690 \mathrm{FP} \\
71 \mathrm{FN} \\
1320 \mathrm{VN} \\
\text { Total } 4079\end{array}$ & $96,6 \%$ & $65,7 \%$ & $74,3 \%$ & $94,9 \%$ & $\begin{array}{l}\text { La méthode de } \\
\text { surveillance en } \\
\text { laboratoire s'est avérée } \\
\text { très sensible, mais peu } \\
\text { spécifique }\end{array}$ \\
\hline
\end{tabular}


Tableau 3 : Études observationnelles-Résumé des statistiques de performance (suite)

\begin{tabular}{|c|c|c|c|c|c|c|c|c|}
\hline \multirow[b]{2}{*}{$\begin{array}{l}\text { Auteur } \\
\text { principal, } \\
\text { année }\end{array}$} & \multirow[b]{2}{*}{ Comparateur } & \multicolumn{6}{|c|}{ Résultats } & \multirow[b]{2}{*}{ Conclusion } \\
\hline & & Taux d'infection & $\begin{array}{l}\text { VP, FP, FN, } \\
\text { VN, Total }\end{array}$ & Sensibilité & Spécificité & $\begin{array}{l}\text { Valeur } \\
\text { prédictive } \\
\text { positive }\end{array}$ & $\begin{array}{l}\text { Valeur } \\
\text { prédictive } \\
\text { négative }\end{array}$ & \\
\hline Rusk, 2016 & $\begin{array}{l}\mathrm{BDCP}+\mathrm{SNISA}+ \\
\mathrm{PCl} \text {, comparées } \\
\text { avec les données } \\
\text { de } \mathrm{PCl} \text { seules }\end{array}$ & $\begin{array}{l}\mathrm{BDCP} / \mathrm{SNISA} / \\
\mathrm{PCl}: 1,7 \text { pour } 100 \\
\text { procédures } \\
\mathrm{PCl}: 1,3 \text { pour } 100 \\
\text { procédures }\end{array}$ & s.o. & $89,9 \%$ & $99 \%$ & s.o. & s.o. & $\begin{array}{l}\text { L'examen des dossiers } \\
\text { médicaux pour les cas } \\
\text { identifiés grâce aux } \\
\text { données administratives } \\
\text { a été une stratégie } \\
\text { efficace pour optimiser la } \\
\text { surveillance de } \mathrm{PCl}\end{array}$ \\
\hline $\begin{array}{l}\text { Daneman, } \\
2011\end{array}$ & $\begin{array}{l}\text { BDCP + SNISA } \\
\text { + déclarations } \\
\text { des médecins } \\
\text { comparées avec } \\
\text { les données de } \\
\text { PCI }\end{array}$ & s.o. & s.o. & $77,3 \%$ & $87 \%$ & $17,4 \%$ & $99,1 \%$ & $\begin{array}{l}\text { Les données } \\
\text { administratives avaient } \\
\text { une sensibilité et } \\
\text { une valeur prédictive } \\
\text { positive faibles et étaient } \\
\text { inadéquates en tant } \\
\text { qu'indicateur de qualité }\end{array}$ \\
\hline $\begin{array}{l}\text { Lethbridge, } \\
2019\end{array}$ & $\begin{array}{l}\text { BDCP ou SNISA } \\
\text { comparées à } \\
\text { BDCP + SNISA + } \\
\text { base de données } \\
\text { des déclarations } \\
\text { des médecins }\end{array}$ & $\begin{array}{l}\text { Différence de } \\
0,44 \text { entre la } \\
\text { BDCP ou le } \\
\text { SNISA seul } \\
\text { et toutes les } \\
\text { données mises } \\
\text { ensemble }\end{array}$ & s.o. & s.o. & s.o. & s.o. & s.o. & $\begin{array}{l}\text { Les taux ont été sous- } \\
\text { estimés en utilisant des } \\
\text { données administratives } \\
\text { de source unique }\end{array}$ \\
\hline
\end{tabular}

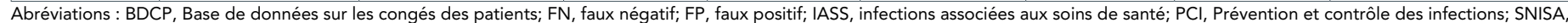
Système national d'information sur les soins ambulatoires; s.o., sans objet; VN, vrai négatif; VP, vrai positif

véritable taux d'incidence des infections. Les résultats montrent que la BDCP inclut les ICD récurrentes et ne peut pas distinguer les cas symptomatiques des asymptomatiques. En fait, la BDCP n'avait qu'une sensibilité modérée de $85 \%$ et une valeur prédictive positive de $80 \%$ (tableau 3).

D'autre part, Daneman et al. ont déterminé si la déclaration publique obligatoire par les hôpitaux était associée à une réduction des taux d'ICD dans les hôpitaux de I'Ontario (23). Outre l'analyse principale, ils ont procédé à une validation croisée des taux d'ICD provenant des données administratives par rapport aux taux déclarés par les établissements individuels via le système de déclaration publique obligatoire. En utilisant des coefficients de corrélation de Pearson pondérés en fonction des jours d'hospitalisation, ils ont pu constater une excellente concordance entre les institutions (23).

Le même coefficient a été utilisé dans l'étude de Ramirez Mendoza et al., (18) qui ont comparé la BDCP avec les données de surveillance relatives aux SARM contractés dans les hôpitaux en Alberta et en Ontario. Les résultats ont montré une forte corrélation entre les données de surveillance de la BDCP et de $\mathrm{PCl}$. L'étude a conclu qu'il y avait là une preuve notable de comparabilité entre ces ensembles de données. Cependant, les taux ou les dénominateurs divergeaient largement entre les données administratives et les données issues de la surveillance active (tableau 3). Certains auteurs n'étaient pas d'accord avec la méthodologie ou la conclusion de l'étude. Notamment, ils étaient en désaccord avec choix de la corrélation de Pearson utilisant des données au niveau de I'hôpital, et avec la conclusion tenant compte de la différence de taux ou de dénominateurs entre les données administratives et les données issues de la surveillance (30).

\section{Études combinant plusieurs bases de données administratives}

Les résultats démontrent que la combinaison des bases de données augmente la précision, sans toutefois atteindre le niveau de précision de la surveillance active traditionnelle. Lethbridge et al. (24) ont combiné plusieurs types de données administratives et les ont comparées à une source unique de données administratives pour cibler les ISO après une arthroplastie de la hanche et du genou en Nouvelle-Écosse. Utilisés seuls, la BDCP et le Système national d'information sur les soins ambulatoires (SNISA) présentaient des taux plus élevés que la base de données des déclarations des médecins, mais sous-estimaient le taux d'infection avec une variation de pourcentage de $44 \%$ par rapport à la combinaison des trois bases de données. Cela implique qu'environ $17 \%$ des cas d'infection auraient été omis si la BDCP ou le SNISA avaient été utilisés seuls. Les auteurs ont conclu que la combinaison des bases de données optimiserait la surveillance des ISO.

Daneman et al. (20) ont évalué la précision de la BDCP, du SNISA et de la base de données des déclarations des médecins par rapport à celle de la surveillance traditionnelle pour la détection des ISO liées à une césarienne survenue en Ontario dans les 30 jours suivant l'intervention chirurgicale. Ils ont établi la sensibilité à seulement 16,7 \% pour la BDCP utilisée seule, à $37,9 \%$ pour la BDCP combinée au SNISA et à $77,3 \%$ pour la BDCP combinée au SNISA et à la base de données des 
déclarations des médecins. Toutes les bases de données ont révélé une spécificité élevée ( $87 \%-98,3 \%)$, mais une valeur prédictive positive très faible $(17,4 \%-27,4 \%$ ) (tableau 3$)$. Les auteurs ont recommandé que les données administratives ne soient pas utilisées comme indicateur de qualité pour la comparaison interhospitalière.

En revanche, Crocker et al. (14) ont comparé les taux d'infection calculés à l'aide d'une combinaison de la BDCP et du SNISA afin de cibler les interventions sur les vertèbres et les ISO. Ils ont démontré que ces taux étaient comparables aux taux d'ISO postopératoires publiés qui ont été recueillis grâce à la surveillance traditionnelle (tableau 3). Cependant, la validité des résultats n'a pas été vérifiée dans cette étude.

\section{Études combinant une base de données administrative et une base de données de laboratoire}

Des études ont démontré que les dossiers de laboratoire pouvaient être utilisés pour améliorer les données administratives. Par exemple, Almond et al. (25) ont évalué la validité d'une méthode de surveillance en laboratoire pour identifier les ICD acquises en milieu hospitalier (ICDAH). Les données de laboratoire seules peuvent entraîner une surestimation des taux d'ICD, un résultat de laboratoire positif ne répondant pas nécessairement aux définitions de cas des ICD acquises en milieu hospitalier (e.g. colonisation asymptomatique, ICD récurrente). Cependant, cette étude a permis d'évaluer la possibilité de lier les résultats d'ICD positifs des laboratoires aux données de la BDCP. L'étude a démontré une sensibilité très élevée, mais une spécificité de $65,7 \%$ et une valeur prédictive positive de $74,3 \%$ (tableau 3 ). Ces résultats indiquent que $26 \%$ des cas classés comme IASS n'étaient pas de véritables cas d'IASS, ce qui signifie qu'un taux plus élevé est observé avec cette méthode. En outre, les auteurs ont effectué une analyse de la fonction d'efficacité du récepteur (courbe $\mathrm{ROC}$ ) pour déterminer si l'utilisation d'un temps entre l'admission et le prélèvement de $\geq 4$ jours était l'algorithme approprié pour classer les cas d'IASS dans l'ensemble des données de laboratoire. L'analyse ROC a indiqué que davantage de cas étaient classés correctement cinq jours après l'admission. Par conséquent, une simple modification dans la définition de cas des ICD classés en tant que IASS pourrait améliorer la spécificité tout en ayant peu d'effet sur la sensibilité.

Une autre étude, celle de Leal et al., est allée plus loin en développant un système de surveillance électronique (SSE) pour le suivi des infections du sang en reliant les bases de données administratives et de laboratoire (21). Le SSE comprend des définitions pour classer les infections du sang et leur lieu de transmission : infection nosocomiale, infection nosocomiale communautaire ou infection communautaire. L'examen des dossiers par le SSE a été comparé à l'examen manuel des dossiers effectué par un assistant de recherche et un médecin spécialiste des maladies infectieuses. L'examen manuel et le SSE ont permis d'identifier 329 et 327 occurrences d'infections du sang, respectivement. Les auteurs ont constaté une grande concordance du lieu de transmission de l'infection (Kappa $=0,78$ ) et ils ont pu ajouter des précisions aux définitions après une révision ultérieure. Les données de surveillance obtenues par le SSE ont permis d'identifier et de classer les infections du sang avec un degré de concordance élevé avec l'examen manuel des dossiers.

\section{Études utilisant des données administratives pour optimiser la surveillance active}

Des études ont montré que les données administratives peuvent être utilisées pour optimiser la surveillance de la $\mathrm{PCl}$. Lee et al. (16) ont évalué les avantages d'établir un lien entre les données représentatives de la surveillance de $\mathrm{PCl}$ et la BDCP pour les cas de SARM acquis à l'hôpital et dans la communauté en Alberta. Cela a permis aux équipes de surveillance de $\mathrm{PCl}$ de disposer d'informations plus pertinentes en temps voulu. Les auteurs ont pu relier avec succès $94,6 \%$ de l'ensemble des dossiers de surveillance et identifier les principales différences entre les patients atteints de SARM acquis à l'hôpital et de SARM acquis dans la communauté, ce qui démontre que les données administratives peuvent être utilisées pour optimiser la surveillance des hôpitaux.

Par l'intermédiaire d'une étude de cohorte rétrospective, Rusk et al. (19) ont évalué une nouvelle stratégie pour améliorer la surveillance traditionnelle de $\mathrm{PCl}$ en utilisant des données administratives pour déclencher l'examen des dossiers médicaux. Les patients admissibles suivis par l'équipe de $\mathrm{PCl}$ ont été associés à la BDCP et au SNISA, et ces bases de données administratives ont fourni les codes de diagnostic et de procédure pour chaque visite ou réadmission. La stratégie visant I'utilisation des données administratives a permis de repérer $87 \%$ des cas identifiés grâce à la surveillance de $\mathrm{PCl}$, avec une sensibilité de $90 \%$ et une spécificité de $99 \%$. Cela a confirmé que l'examen des dossiers médicaux déclenché grâce aux données administratives est une stratégie efficace pour optimiser la surveillance des ISO.

\section{Étude visant à améliorer la comparaison des hôpitaux à l'aide de données administratives}

Daneman et al. (22) ont démontré que les données administratives (BDCP + déclarations des médecins) peuvent être utilisées pour créer un indice de stratification du risque chirurgical modifié pour la surveillance des infections nosocomiales, comparable à celui utilisé pour la surveillance clinique. Cet indice a permis d'ajuster le taux d'infection établi lors de la comparaison avec d'autres établissements. L'étude conclut que les sources de données administratives comme les sources de données cliniques peuvent contribuer à la surveillance des infections, puisque les données administratives peuvent être 
utilisées pour identifier les patients présentant des infections potentielles, ou pour permettre une meilleure détection des diagnostics après le congé du patient.

\section{Revue systématique et données administratives}

Une seule étude (26) a évalué la précision des données administratives pour la surveillance des IASS. Les autres faisaient l'examen d'articles sur les SSE en utilisant les dossiers médicaux électroniques pour la surveillance des IASS et en les comparant à la surveillance traditionnelle, tout en incluant de nombreux articles où une combinaison de données administratives et un SSE avait été utilisée (27-29). Les données administratives se sont avérées avoir une sensibilité et une valeur prédictive positive très hétérogènes, généralement de niveau faible à modeste, avec une précision particulièrement faible pour l'identification des IASS associées à des dispositifs (e.g. infections du système sanguin associées aux cathéters centraux, les infections des voies urinaires associées aux cathéters) (tableau 4) $(26,28)$. En général, la précision très variable des données administratives était principalement due à la quantité de codes de diagnostic différents utilisés entre les études (26). Van Mourik et al. ont évalué l'exactitude des données administratives. Un tiers des études incluses dans la revue présentaient des limites méthodologiques importantes, et celles qui présentaient un risque de biais plus élevé étaient associées à un tableau plus optimiste que celles qui utilisaient des méthodologies strictes (26). En revanche, Leal et al. ont constaté une bonne sensibilité et une excellente spécificité pour les données administratives (tableau 5) (29). Cependant, les populations et les méthodologies dans les différentes études étaient très hétérogènes, et la qualité des études n'a pas été évaluée. Les quatre revues ont montré que la combinaison des sources de données administratives avec d'autres sources de surveillance, en particulier avec des données de microbiologie, améliorait la précision. Des études ont également démontré que les données de microbiologie avaient une bonne sensibilité $(28,29)$; cependant, Freeman et al. ont conclu que les SSE utilisant uniquement des données de microbiologie avaient tendance à surestimer les taux d'IASS (27). Streefkerk et al. (28) ont également constaté que les données de microbiologie combinées à la prescription d'antibiotiques et aux données de laboratoire (biochimie, hématologie, etc.) étaient plus précises que la microbiologie seule (tableau 5). Enfin, la plupart des études ont conclu que les données administratives étaient avantageuses pour suivre les IASS nécessitant une surveillance après le congé du patient (e.g. les ISO).

\section{Revue systématique et système de surveillance électronique}

Les résultats ont démontré que la surveillance électronique à l'aide d'algorithmes de détection des IASS à partir des dossiers médicaux électroniques n'avait pas encore atteint son plein potentiel, mais qu'elle présentait des possibilités intéressantes

\section{Tableau 4 : Revue systématique-Résumé des statistiques de performance par type d'infection associée aux soins} de santé

\begin{tabular}{|c|c|c|c|c|c|c|c|}
\hline \multirow{2}{*}{$\begin{array}{l}\text { Auteur } \\
\text { principal, } \\
\text { année }\end{array}$} & \multirow{2}{*}{$\begin{array}{c}\text { Nombre } \\
\text { d'articles inclus }\end{array}$} & \multicolumn{5}{|c|}{$\begin{array}{c}\text { Sensibilité (SE), Spécificité (SP), Valeur prédictive positive (VPP), Valeur prédictive } \\
\text { négative (VPN) }\end{array}$} & \multirow{2}{*}{$\begin{array}{l}\text { Autres } \\
\text { renseignements }\end{array}$} \\
\hline & & ISO & IS/ISSACC & ICD & Pneumonie/PAV & IVU/IVUAC & \\
\hline $\begin{array}{l}\text { Freeman, } \\
2013\end{array}$ & $\begin{array}{l}n=44 \\
(I S O=6 \\
I S=11 \\
I V U=4 \\
\text { Pneumonie = } 4 \\
\text { Autres }=8 \\
\text { IASS multiples } \\
=12 \text { ) }\end{array}$ & $\begin{array}{l}\mathrm{SE}=60 \%-98 \% \\
\mathrm{SP}=91 \%-99 \%\end{array}$ & $\begin{array}{l}\mathrm{SE}=72 \%-100 \% \\
\mathrm{SP}=37 \%-100 \%\end{array}$ & $\begin{array}{l}\mathrm{SE}=80 \%-83 \% \\
\mathrm{SP}=99,9 \%\end{array}$ & $\begin{array}{l}\mathrm{SE}=71 \%-99 \% \\
\mathrm{SP}=61 \%-100 \%\end{array}$ & $\begin{array}{l}\mathrm{SE}=86 \%-100 \% \\
\mathrm{SP}=59 \%-100 \%\end{array}$ & $\begin{array}{l}\text { Trois études ont } \\
\text { utilisé des données } \\
\text { provenant d'une } \\
\text { seule source, } 37 \text { ont } \\
\text { utilisé des données } \\
\text { provenant de plusieurs } \\
\text { sources, dont les } \\
\text { laboratoires, et } \\
\text { quatre ont utilisé des } \\
\text { données provenant } \\
\text { de plusieurs } \\
\text { sources, excluant les } \\
\text { laboratoires }\end{array}$ \\
\hline $\begin{array}{l}\text { Van Mourik, } \\
2015\end{array}$ & $\begin{array}{l}\mathrm{n}=57 \\
(\mathrm{ISO}=34 \\
\mathrm{IS}=24 \\
\text { Pneumonie = } 14 \\
\mathrm{IVU}=15 \\
\text { Autre }=7)\end{array}$ & $\begin{array}{l}\mathrm{SE}=10 \%-100 \% \\
\mathrm{VPP}=11 \%-95 \%\end{array}$ & $\begin{array}{l}\text { ISSACC } \\
\text { - Sensibilité } \\
\text { inférieure à } 40 \% \\
\text { pour toutes les } \\
\text { études sauf une } \\
\text { - SE plus élevé pour } \\
\text { IS/sepsie }\end{array}$ & - & $\begin{array}{l}\text { Pneumonie } \\
\text { SE et PPV environ } \\
40 \% \\
\text { PAV } \\
\text { SE }=37 \%-72 \% \\
\text { PPV }=12 \%-57 \%\end{array}$ & $\begin{array}{l}\text { SE inférieur à } 60 \% \\
\text { PPV inférieur à } \\
25 \% \\
\text { SE plus élevé en } \\
\text { cas d'IVU qu'en } \\
\text { cas d'IVUACC }\end{array}$ & $\begin{array}{l}\text { Gain de sensibilité } \\
\text { de près de } 10 \% \text { en } \\
\text { combinant les bases } \\
\text { de données } \\
\text { Les études présentant } \\
\text { un risque de biais plus } \\
\text { élevé étaient plus } \\
\text { optimistes }\end{array}$ \\
\hline $\begin{array}{l}\text { Streefkerk, } \\
2020\end{array}$ & $\begin{array}{l}n=78 \\
(I S O=29 \\
I S=33 \\
\text { Pneumonie = 16 } \\
\text { IVU = 18) }\end{array}$ & $\begin{array}{l}\mathrm{SE}=0,02-1,0 \\
\mathrm{SP}=0,59-1,0\end{array}$ & $\begin{array}{l}\mathrm{SE}=0,32-1,0 \\
\mathrm{SP}=0,37-1,0\end{array}$ & - & $\begin{array}{l}\mathrm{SE}=0,33-1,0 \\
\mathrm{SP}=0,58-1,0\end{array}$ & $\begin{array}{l}\mathrm{SE}=0,02-1,0 \\
\mathrm{SP}=0,59-1,0\end{array}$ & $\begin{array}{l}\text { La sensibilité était } \\
\text { généralement élevée, } \\
\text { mais la spécificité très } \\
\text { variable }\end{array}$ \\
\hline
\end{tabular}

Abréviations : ICD, infection à C. difficile; IS, infection du sang; ISO, infection du site opératoire; ISSACC, infections du système sanguin associées aux cathéters centraux; IVU, infection des voies urinaires; IVUAC, infection des voies urinaires associée aux cathéters; PAV, pneumonie associée à la ventilation assistée; -, aucun résultat présenté dans cette étude 
Tableau 5 : Revue systématique—Résumé des statistiques de performance par type de surveillance

\begin{tabular}{|c|c|c|c|c|c|c|c|c|}
\hline \multirow{3}{*}{$\begin{array}{l}\text { Auteur } \\
\text { principal, } \\
\text { année }\end{array}$} & \multirow{3}{*}{$\begin{array}{l}\text { Nombre } \\
\text { d'articles } \\
\text { inclus }\end{array}$} & \multicolumn{6}{|c|}{ Sensibilité (SE), Spécificité (SP) (portée ou moyenne) } & \multirow[b]{3}{*}{$\begin{array}{c}\text { Autres } \\
\text { renseignements }\end{array}$} \\
\hline & & \multirow[b]{2}{*}{$\begin{array}{l}\text { Données } \\
\text { administratives }\end{array}$} & \multicolumn{3}{|c|}{ laboratoire } & \multirow[b]{2}{*}{$\begin{array}{l}\text { Données } \\
\text { administratives } \\
\text { + données de } \\
\text { laboratoire }\end{array}$} & \multirow[b]{2}{*}{ Autre } & \\
\hline & & & Microbiologie & $\begin{array}{l}\text { Microbiologie } \\
\text { + prescription } \\
\text { d'antibiotiques }\end{array}$ & $\begin{array}{c}\text { Microbiologie } \\
+ \text { prescription } \\
\text { d'antibiotiques } \\
+ \text { chimie }\end{array}$ & & & \\
\hline $\begin{array}{l}\text { Streefkerk, } \\
2020\end{array}$ & $\begin{array}{l}n=78 \\
(D A=7, \\
L=61 \\
A=10)\end{array}$ & $\begin{array}{l}\mathrm{SE}=30 \%^{\mathrm{a}} \\
\mathrm{SP}=94,5 \%^{\mathrm{a}}\end{array}$ & $\begin{array}{l}S E=77 \% \\
S P=92 \%\end{array}$ & $\begin{array}{l}S E=92 \% \\
S P=86 \%\end{array}$ & $\begin{array}{l}S E=93 \% \\
S P=94 \%\end{array}$ & - & $\begin{array}{l}S E=86 \% \\
S P=90 \%\end{array}$ & $\begin{array}{l}\text { En général, la } \\
\text { sensibilité des études } \\
\text { utilisant des données } \\
\text { de microbiologie est } \\
\text { bonne }\end{array}$ \\
\hline Leal, 2008 & $\begin{array}{l}n=24 \\
(D A=7, \\
L=6, \\
D A+ \\
L=6, \\
A=5)\end{array}$ & $\begin{array}{l}\mathrm{SE}=59 \%-96 \%^{\mathrm{b}} \\
\mathrm{SP}=95 \%-99 \%^{\mathrm{b}}\end{array}$ & $\begin{array}{l}\mathrm{SE}=63 \%-91 \% \\
\mathrm{SP}=87 \%-99 \%\end{array}$ & & & $\begin{array}{l}\mathrm{SE}=71 \%-95 \% \\
\mathrm{SP}=47 \%-99 \%\end{array}$ & - & $\begin{array}{l}\text { L'association DA + } \\
\text { L a donné lieu à une } \\
\text { SE plus élevée, mais } \\
\text { à une SP plus faible } \\
\text { que pour l'une et } \\
\text { l'autre des sources } \\
\text { lorsqu'utilisée seule }\end{array}$ \\
\hline
\end{tabular}

Abréviations : $A$, autre; $D A$, étude de données administratives; $L$, étude de données de laboratoire; -, aucun résultat présenté dans cette étude

a Codage de la classification internationale des maladies (CIM) uniquement

${ }^{b}$ Codage de la classification internationale des maladies (CIM) + pharmacie + bases de données des déclarations des médecins

et un bon potentiel. La plupart des études ont conclu que des SSE devraient être développés et utilisés dans les hôpitaux, tout en reconnaissant que ces méthodes peuvent réduire la charge associée à la surveillance manuelle traditionnelle (27-29). En fait, la sensibilité était généralement élevée et la spécificité variable pour la plupart des SSE, en comparaison avec la surveillance active traditionnelle (tableaux 4 et 5). Freeman et al. ont constaté que de nombreux algorithmes informatiques de surveillance électronique étaient plus performants que la méthode d'examen manuel des dossiers (27). La majorité des études de cette revue ont insisté sur le couplage des bases de données électroniques avec un système de surveillance " interne ", plutôt qu'avec un logiciel commercial (27). Streefkerk et al. ont démontré que le meilleur SSE utilisait une procédure en deux étapes, soit la sélection des cas par le système, suivie d'une évaluation de confirmation des cas sélectionnés par l'équipe de $\mathrm{PCl}$ (28). Dans la même revue, sept études visaient à développer un SSE capable de détecter toutes les IASS, avec une sensibilité allant de 0,78 à 0,99 . Leal et al. ont démontré que les SSE étaient potentiellement peu coûteux, efficaces et pouvaient atteindre une sensibilité de $100 \%$ lorsque l'infection visée pouvait être définie par la présence d'une culture positive (29). Cependant, les SSE étaient moins efficaces lorsque l'infection ne pouvait être diagnostiquée que sur la base d'une évaluation clinique des symptômes, ou de tests autres qu'une culture microbiologique positive. En outre, la qualité des données et du couplage peut influencer la qualité du SSE (29). Freeman et al. ont également conclu que dans certaines études, l'absence de données cliniques dans un format électronique réduisait la capacité du SSE à détecter les IASS (27).

\section{Analyse}

Le Canada dispose de données administratives sur la santé d'une grande richesse, lesquelles sont recueillies au niveau provincial et territorial auprès des diverses institutions composant le système de soins de santé. Cependant, ces données ne sont pas utilisées à leur plein potentiel et leur utilisation accrue pourrait améliorer les efforts de surveillance des IASS, en plus de réduire la charge de travail associée à la surveillance active traditionnelle. Cet examen de la portée a considéré l'utilisation et la validité des données administratives, utilisées seules ou combinées à d'autres sources de données, pour la surveillance des IASS au Canada. Dans l'ensemble, les études ont révélé que I'utilisation d'une seule source de données administratives pour la surveillance des IASS ne donne pas des résultats suffisamment précis par rapport à la surveillance active traditionnelle. Cependant, la combinaison de différentes sources de données permet d'accroître la précision. De plus, la combinaison des données administratives et de la surveillance active s'est avérée être une stratégie efficace pour optimiser la surveillance active et ainsi réduire la charge de travail des équipes de $\mathrm{PCl}$.

\section{Avantages et inconvénients des données administratives}

Les données administratives ne sont pas collectées à des fins de surveillance. Cependant, elles présentent de nombreuses caractéristiques intéressantes qui en font une solution potentielle pour l'optimisation de la surveillance des IASS. Elles sont peu coûteuses, disponibles dans presque tous les établissements de santé, recueillies de manière cohérente, soumises à un contrôle de qualité et ne représentent pas une charge administrative supplémentaire pour les cliniciens ou les patients (31). Des couplages déterministes peuvent également être établis avec les bases de données qui recueillent les numéros d'assurance-maladie, puisque chaque Canadien possède un numéro unique.

En outre, de nombreuses études ont démontré que les données administratives sont avantageuses pour le suivi 
des IASS nécessitant une surveillance après le congé du patient $(19,20,22,26)$. Ceci est particulièrement important pour les infections telles que les ISO, dont la majorité se développe après que le patient ait quitté I'hôpital (19,32-34). Par exemple, dans l'étude de Rusk et al., on mentionne que $96 \%$ des cas d'ISO ont été identifiés après la sortie du patient de l'hôpital, et que $43 \%$ des cas d'ISO confirmés ont été identifiés dans un établissement autre que celui où la procédure avait été effectuée (19). Ces résultats confirment que si la surveillance active des ISO est effectuée uniquement à I'hôpital où se déroule l'opération, la détection des ISO est limitée. Les pratiques exemplaires en matière de surveillance des infections associées aux soins de santé publiées par Santé publique Ontario indiquent que « jusqu'à présent, il n'existe aucune méthode généralement acceptée de surveillance d'après congé pour les ISO à l'extérieur du centre hospitalier [...] Pour détecter les ISO survenant après le congé, il est préférable que les professionnels en contrôle des infections conçoivent des méthodes novatrices qui n'empiètent pas sur le temps consacré aux autres composantes du système de surveillance » (35). Parmi les exemples de solutions proposées, citons I'utilisation de bases de données administratives et la détection électronique des symptômes et des signes d'infection dans les dossiers des patients après leur congé (35).

\section{Obstacles à l'obtention de données administratives précises pour la surveillance des infections associées aux soins de santé au Canada}

Au Canada, I'ICIS recueille des données cliniques par I'intermédiaire des bases de données clinico-administratives, qui se composent de deux bases de données distinctes: La Base de données sur la morbidité hospitalière, générée à partir de la BDCP, et le SNISA (36). À I'heure actuelle, I'ICIS rapporte publiquement certaines IASS, comme les sepsies contractées à l'hôpital, les infections des voies urinaires et les organismes résistants aux antimicrobiens, la plupart au niveau national seulement, à l'aide des données recueillies grâce à la BDCP. L'ICIS dispose d'un programme complet d'assurance de la qualité des données. Tous les problèmes de qualité connus sont traités par le fournisseur de données, ou consignés dans la documentation spécifique aux limites des données mise à la disposition de tous les utilisateurs (36). Cependant, il reste de nombreux obstacles à surmonter avant de pouvoir produire des données administratives précises pour la surveillance des IASS. Les études confirment que le manque de précision est une limite importante à l'utilisation des données administratives comme indicateur de la qualité pour la comparaison des hôpitaux. Par exemple, la variabilité de la pratique médicale, la documentation et le codage relatifs aux sommaires d'hospitalisation entre les établissements, I'interprétation faite par les professionnels du codage médical, le fait que la collecte des données repose sur les prestataires de soins primaires et que les informations sont basées sur leur capacité à détecter et à signaler une IASS (erreurs possibles de classification, erreurs humaines) $(15,19,37,38)$. L'information est principalement limitée par le contenu du dossier médical et dépend principalement de la consignation adéquate des renseignements par le clinicien.

Par exemple, la transmission à la base de données BDCP exige que le médecin remplisse de manière adéquate le sommaire d'hospitalisation du patient en y incluant les IASS, si elles sont connues. Les IASS ne sont généralement pas détectées en temps réel et risquent d'être évaluées de façon différente par un clinicien et par l'équipe de prévention et de contrôle des infections, étant donné que cette dernière suit des définitions standardisées. Un spécialiste du codage professionnel du service des dossiers médicaux traduit ensuite les sommaires d'hospitalisation en codes standard. Dans le cadre d'une étude menée en 2015-2016 en Alberta, des professionnels du codage ont été interrogés au sujet des obstacles qui nuisent à la production de données administratives de haute qualité et qui sont le fait des médecins (39). Ces obstacles englobent une consignation incomplète et non spécifique des renseignements de la part des médecins, l'utilisation d'une terminologie différente par les médecins et par les codeurs (e.g. le diagnostic du médecin ne figure pas dans la liste de la $\mathrm{CIM}-10)$, le manque de communication entre les codeurs et les médecins (principalement en milieu urbain) et le fait que les codeurs soient limités dans leur capacité à compléter, modifier ou interpréter les informations consignées par le médecin. Enfin, les codeurs ne sont pas autorisés à utiliser des documents justificatifs qui pourraient accroître la spécificité des codes de diagnostic (e.g. rapports de laboratoire) (39). En fait, un obstacle de taille pour l'ICIS est que la consignation des informations par le médecin a généralement priorité sur toute autre documentation, même si les rapports de laboratoire ou $d$ 'autres documents indiquent un diagnostic différent. Pourtant, de nombreuses études démontrent que les données de laboratoire pourraient être utilisées pour améliorer les données administratives $(13,21,29,37)$. Ainsi, permettre aux codeurs d'utiliser les données de laboratoire pourrait être une solution envisageable pour améliorer la précision du codage.

\section{Intégration des données administratives dans la surveillance effectuée par les équipes de prévention et de contrôle des infections}

Des études ont également démontré que l'utilisation de données administratives par l'équipe de $\mathrm{PCl}$ peut réduire sa charge de travail et améliorer la surveillance des IASS. Lee et al. ont illustré que le fait de coupler les données de surveillance aux données administratives permet de disposer d'informations détaillées en temps opportun, et ils ont exhorté les juridictions et les systèmes de santé à envisager d'adopter ce type de couplage de données au bénéfice des pratiques de surveillance (16). Rusk et al. ont confirmé l'existence d'une stratégie efficace pour identifier les cas potentiels d'ISO en vue d'un examen plus approfondi par l'équipe de $\mathrm{PCl}$ grâce à l'utilisation des codes de données 
administratives. Cela améliorerait la cohérence de la recherche de cas et réduirait le temps et les ressources nécessaires (19). Toutes ces études ont prouvé que les données administratives peuvent être utilisées pour optimiser la surveillance traditionnelle effectuée par l'équipe de $\mathrm{PCl}$. L'inverse pourrait également être vrai. Comme indiqué précédemment, les codeurs ne peuvent utiliser que les renseignements consignés par les médecins pour déclarer les diagnostics. D'autre part, la surveillance traditionnelle effectuée par les professionnels de $\mathrm{PCl}$ est considérée comme la référence en matière de surveillance, et produit des données précises. Si les codeurs pouvaient accéder aux résultats de la surveillance effectuée par les équipes de $\mathrm{PCl}$, cela pourrait renforcer la validité de la consignation des renseignements par le médecin et l'interprétation des codeurs.

\section{Intégration des données administratives dans les systèmes de surveillance électronique}

Une autre approche potentielle pour rendre la surveillance moins laborieuse consiste à utiliser des systèmes de surveillance électronique. Sept études observationnelles présentées dans le cadre du présent examen ont évalué le couplage des données de bases électroniques, tandis que trois revues systématiques ont analysé les systèmes de surveillance électronique. Leal et al. ont développé un SSE complet pour identifier et classer les infections du sang tout en respectant un haut degré de concordance avec les résultats obtenus par l'examen manuel des dossiers (21). Les conclusions de la revue systématique de Freeman et al. suggèrent que la création d'un SSE est réalisable dans de nombreux contextes et qu'il devrait être développé par les hôpitaux (27). Le SSE peut également être développé de manière à pouvoir cibler plus d'une IASS. En outre, la revue systématique de Steefkerk et al. sur les SSE a présenté les 10 meilleures études, sélectionnées en fonction de leur qualité et de leur performance. Pour la majorité d'entre elles, une procédure incluant deux étapes a été utilisée, soit la sélection de données administratives, de dossiers médicaux électroniques ou de données de microbiologie par le SSE, suivie d'une confirmation par un professionnel de $\mathrm{PCl}$ (28). Dans ce type de cas, le SSE pourrait être conçu pour privilégier la sensibilité par rapport à la spécificité, sachant que l'examen manuel exclura les faux positifs (31). Streefkerk et al. ont présenté sept études portant sur des SSE capables de cibler toutes les IASS (28). Leur revue a même inclus une étude décrivant un algorithme très performant pour détecter les IASS en temps réel, doté d'une sensibilité de $99 \%$ et une spécificité de $93 \%$. Les IASS visés incluaient les infections des voies urinaires, les infections du sang, les infections des voies respiratoires, les infections du tube digestif, les infections de la peau et des tissus mous et d'autres infections (parotidite, varicelle, infections neurologiques, etc.) (40). Toutefois, ces sept études n'ont pas été réalisées au Canada. En fait, les établissements d'autres pays disposent déjà de données électroniques et la mise en œuvre d'un SSE pour la surveillance des IASS est donc plus facilement réalisable. Au Canada, tous les hôpitaux n'ont pas encore accès à un système efficace de gestion des dossiers médicaux électroniques.
Certaines provinces sont de bons modèles pour la surveillance grâce aux données électroniques. Par exemple, la plupart des études incluses dans cet examen de la portée provenaient de provinces possédant des systèmes électroniques (e.g. Alberta, Ontario). L'Alberta est un bon exemple de surveillance des IASS, car toutes les institutions de soins de courte durée effectuent une surveillance traditionnelle en utilisant un protocole de surveillance unique et un système centralisé de saisie des données en ligne (41). Ce système permet de partager les données administratives entre toutes les installations qui l'utilisent. Le Québec dispose également d'un système électronique centralisé créé pour le programme Surveillance provinciale des infections nosocomiales. Ce système utilise des définitions uniformes pour cibler les IASS (42); toutefois, aucune étude québécoise ne répondait à nos critères d'inclusion. Une étude de Gilca et al. mérite d'être prise en considération : cette étude portait sur 83 hôpitaux offrant des soins de courte durée et participant à la surveillance des ICD dans la province de Québec (43). Les auteurs ont comparé les données administratives et les données issues de la surveillance et ont constaté une excellente concordance entre les taux obtenus à partir de MED-ÉCHO (base de données sur les congés des patients) et l'incidence des ICD calculée selon la surveillance provinciale. Cependant, l'origine de la transmission des ICD n'était pas indiquée dans la base de données administrative. II n'a donc pas été possible de distinguer les cas nosocomiaux des cas acquis dans la communauté uniquement grâce aux données administratives.

Une étude menée dans trois états des États-Unis ainsi que dans la province de l'Ontario, au Canada, a évalué les difficultés en matière de technologie de l'information et les stratégies imaginées pour développer et mettre en œuvre un système électronique multihospitalier, dans le but de prévenir les SARM (44). L'étude visait 11 hôpitaux, tous dotés d'un groupe des technologies de l'information (TI) en sous-effectif et de sept systèmes différents, tous ayant une infrastructure de $\mathrm{Tl}$ et un système de données uniques. Les auteurs ont imaginé des stratégies innovantes pour permettre la collecte, le partage, l'analyse et la communication automatisés des données, et ce, dans un format compatible pour tous les hôpitaux. L'étude a été publiée en 2013, et les auteurs appliquent actuellement les mêmes stratégies pour développer des SSE ciblant d'autres IASS. Cette étude est un bon exemple de la faisabilité des SSE malgré l'utilisation de différents systèmes au niveau hospitalier.

\section{Forces et faiblesses}

Nous avons utilisé des méthodes standardisées et rigoureuses pour identifier la littérature publiée, l'examiner et en évaluer la qualité. Toutes ces étapes ont été réalisées par deux examinateurs indépendants. Deux stratégies de recherche différentes ont été utilisées, afin d'assurer que toutes les études canadiennes soient incluses, ainsi que les revues systématiques incluant au moins une étude menée au Canada. Notre examen a porté sur un petit nombre d'études; toutefois, nous sommes convaincus que nos stratégies de recherche combinées à la 
recherche manuelle ont permis de repérer tous les articles pertinents disponibles. Il s'agit du premier examen de la portée à rendre compte des divergences entre les données administratives et les données issues de la surveillance utilisées dans le cadre de la surveillance des IASS au Canada.

Celui-ci, cependant, présente plusieurs limites. Nous n'avons inclus que des études qui ont été publiées en français ou en anglais. Par contre, puisque le français et l'anglais sont les deux langues officielles du Canada, nous ne pensons pas avoir négligé d'autres études importantes. Les études observationnelles sélectionnées ne représentent que trois provinces canadiennes, les deux tiers des études provenant de l'Alberta. L'Alberta dispose d'un système de santé intégré à l'échelle de la province qui est accessible et facile à consulter, ce qui n'est pas le cas pour les systèmes utilisés dans les autres provinces. Bien que notre examen ait porté sur des articles publiés en anglais ou en français, nous avons mené notre recherche en utilisant uniquement des termes anglais. Nous n'avons effectué nos recherches qu'à partir de trois bases de données; ainsi, peut-être sommes-nous passés à côté d'articles pertinents inclus dans d'autres bases de données. Cette étude a été menée sur des données canadiennes uniquement et peut ne pas être représentative pour d'autres pays.

\section{Conclusion}

Cet examen de la portée a permis de constater les nombreuses divergences entre les données administratives et les données issues de la surveillance active utilisées dans le cadre de la surveillance des IASS dans les hôpitaux canadiens. Cependant, elle nous a également permis d'identifier des solutions potentielles en fonction de l'IASS surveillée, tout en démontrant que les données administratives peuvent être utilisées pour optimiser la surveillance des IASS. Les systèmes de surveillance électronique peuvent permettre d'économiser du temps et des ressources humaines et la combinaison de plusieurs ensembles de données administratives peut également améliorer la précision des données. L'équipe de $\mathrm{PCl}$ qui a fait usage de données administratives ou de systèmes de surveillance électronique a pu réduire sa charge de travail quant à la surveillance active. Bien que la surveillance active des IASS ait produit les résultats les plus précis et demeure la norme d'excellence, d'autres études sur la surveillance des IASS au Canada devraient porter sur la faisabilité du partage des données entre les provinces grâce à des systèmes électroniques, ainsi sur la possibilité pour les professionnels du codage médical d'accéder à une documentation autre que les seules déclarations des médecins, puis sur la possibilité d'utiliser des données administratives pour aider à réduire le fardeau de la surveillance active.

\section{Déclaration des auteurs}

V. B. - Conceptualisation, méthodologie, investigation, validation, analyse formelle, rédaction-projet original

E. P. - Investigation, validation, rédaction-révision

A. M. - Conceptualisation, ressources, rédaction-révision et édition, acquisition de fonds

C. Q. - Conceptualisation, rédaction-révision et édition, supervision, acquisition de fonds

Le contenu de l'article et les points de vue qui y sont exprimés n'engagent que les auteurs et ne correspondent pas nécessairement à ceux du gouvernement du Canada.

\section{Intérêts concurrents}

Aucun des auteurs n'avait de conflits d'intérêts à divulguer.

\section{Remerciements}

V. Boulanger et $\mathrm{E}$. Poirier sont soutenus grâce à un stage de MITACS Accélération - Excellence en santé Canada. Nous remercions $\mathrm{M}$. Clar pour son aide au niveau de la stratégie de recherche documentaire. L. Prelude et $A$. Chapman pour leur soutien, ainsi que D. Diallo pour son aide à la traduction anglaise. C. Quach est titulaire d'une chaire de recherche du Canada de niveau 1 dans le domaine de la prévention des infections, de I'hôpital à la communauté.

\section{Financement}

Ce travail a été financé par Excellence en santé Canada et MITACS Accélération - Excellence en santé Canada.

\section{Matériel supplémentaire}

Ces documents sont accessibles dans le dossier

Données supplémentaires

Données supplémentaires S1

Données supplémentaires S2

Tableau supplémentaire S3

Tableau supplémentaire S4

\section{Références}

1. Programme canadien de surveillance des infections nosocomiales. Les infections associées aux soins de santé et la résistance aux antimicrobiens dans les hôpitaux canadiens de soins de courte durée, 2014 à 2018. Relevé des maladies transmissibles au Canada 2020;46(5):111-26. DOI 
2. MacLaurin A, Amaratunga $K$, Couris $C$, Frenette $C$, Galioto $R$, Hansen G, Happe J, Neudorf K, Pelude L, Quach C, Rose SR. Measuring and Monitoring Healthcare-Associated Infections: A Canadian Collaboration to Better Understand the Magnitude of the Problem. Healthc Q. 2020;22(SP):116-28. DOl PubMed

3. Mitchell R, Taylor G, Rudnick W, Alexandre S, Bush K, Forrester L, Frenette C, Granfield B, Gravel-Tropper D, Happe J, John M, Lavallee C, McGeer A, Mertz D, Pelude L, Science M, Simor A, Smith S, Suh KN, Vayalumkal J, Wong A, Amaratunga K; Canadian Nosocomial Infection Surveillance Program. Trends in health care-associated infections in acute care hospitals in Canada: an analysis of repeated point-prevalence surveys. CMAJ 2019;191(36):E981-8. DOI PubMed

4. Aiello R. PM Trudeau questioned on COVID-19 projections, implores people to stay home. Ottawa News Bureau Online Producer, CTV News, April 2, 2020. https://www.ctvnews. $\mathrm{ca} /$ health/coronavirus/pm-trudeau-questioned-on-covid-19projections-implores-people-to-stay-home-1.4879062? cache= sgjigezwskldpu\%3FcontactForm\%3Dtrue

5. Agence de la santé publique du Canada Le programme canadien de surveillance des infections nosocomiales (PCSIN) : Rapport sommaire sur les données de surveillance des infections associées aux soins de santé (IASS), la résistance aux antimicrobiens (RAM) et l'utilisation des antimicrobiens (UAM) du 1er janvier 2013 au 31 décembre 2017. Ottawa (ON) : ASPC (modifié 2021-07). https://www.canada.ca/en/ public-health/services/publications/science-research-data/ summary-report-healthcare-associated-infection-antimicrobialresistance-antimicrobial-use-surveillance-data-2013-2017.html

6. Ruthledge-Taylor K, Mitchell R, Prelude L, AbdelMalik P, Roth V. Evaluation of the representativeness of the Canadian Nosocomial Infection Surveillance Program. Can J Infect Control 2015;30(1):13-7. https://ipac-canada.org/ photos/custom/OldSite/cjic/vol30no1.pdf

7. Xia $\mathrm{Y}$, Tunis $\mathrm{MC}$, Frenette $\mathrm{C}$, Katz $\mathrm{K}$, Amaratunga $\mathrm{K}$, Rhodenizer Rose S, House A, Quach C. Épidémiologie de I'infection Clostridioides difficile au Canada : revue de six ans de données en appui au processus décisionnel quant à I'utilisation des vaccins. Relevé des maladies transmissibles au Canada 2019;45(7/8):211-32. DOI

8. Quan H, Smith M, Bartlett-Esquilant G, Johansen H, Tu K, Lix L; Hypertension Outcome and Surveillance Team. Mining administrative health databases to advance medical science: geographical considerations and untapped potential in Canada. Can J Cardiol 2012;28(2):152-4. DOI PubMed

9. Canadian Institute for Health Information. CIHI Data Quality Study of the 2009-2010 Discharge Abstract Database. Ottawa (ON): CIHI; 2012. https://secure.cihi.ca/free_products/ Reab\%202009-2010\%20Main\%20Report\%20FINAL.pdf

10. Willemse C. Using the Canadian Institute for Health Information's Information Quality Framework to Support Integration and Utilization of Complex, Multi-Jurisdictional Data. Internat J Pop Data Sci. 2020;5(5). https://ijpds.org/ article/view/1556
11. Tricco $A C$, Lillie $E$, Zarin $W, O^{\prime} B$ rien $K K$, Colquhoun $H$, Levac D, Moher D, Peters MD, Horsley T, Weeks L, Hempel S, Akl EA, Chang C, McGowan J, Stewart L, Hartling L, Aldcroft A, Wilson MG, Garritty C, Lewin S, Godfrey CM, Macdonald MT, Langlois EV, Soares-Weiser K, Moriarty J, Clifford T, Tunçalp Ö, Straus SE. PRISMA Extension for Scoping Reviews (PRISMA-ScR): checklist and Explanation. Ann Intern Med 2018;169(7):467-73. DOI PubMed

12. Sterne JA, Hernán MA, Reeves BC, Savović J, Berkman ND, Viswanathan M, Henry D, Altman DG, Ansari MT, Boutron I, Carpenter JR, Chan AW, Churchill R, Deeks JJ, Hróbjartsson A, Kirkham J, Jüni P, Loke YK, Pigott TD, Ramsay CR, Regidor $D$, Rothstein HR, Sandhu L, Santaguida PL, Schünemann HJ, Shea B, Shrier I, Tugwell P, Turner L, Valentine JC, Waddington $\mathrm{H}$, Waters E, Wells GA, Whiting PF, Higgins JP. ROBINS-I: a tool for assessing risk of bias in non-randomised studies of interventions. BMJ 2016;355:i4919. DOI PubMed

13. Shea BJ, Reeves BC, Wells G, Thuku M, Hamel C, Moran J, Moher D, Tugwell P, Welch V, Kristjansson E, Henry DA. AMSTAR 2: a critical appraisal tool for systematic reviews that include randomised or non-randomised studies of healthcare interventions, or both. BMJ 2017;358:j4008.

DOI PubMed

14. Crocker A, Kornilo A, Conly J, Henderson E, Rennert-May $\mathrm{E}$, Leal J. Using administrative data to determine rates of surgical site infections following spinal fusion and laminectomy procedures. Am J Infect Control 2021;49(6):759-63. DOI PubMed

15. Pfister T, Rennert-May E, Ellison J, Bush K, Leal J. Clostridioides difficile infections in Alberta: the validity of administrative data using ICD-10 diagnostic codes for CDI surveillance versus clinical infection surveillance. Am J Infect Control 2020;48(12):1431-6. DOI PubMed

16. Lee S, Ronksley P, Conly J, Garies S, Quan H, Faris P, Li B, Henderson E. Using data linkage methodologies to augment healthcare-associated infection surveillance data. Infect Control Hosp Epidemiol 2019;40(10):1144-50.DOI PubMed

17. Rennert-May E, Manns B, Smith S, Puloski S, Henderson E, Au F, Bush K, Conly J. Validity of administrative data in identifying complex surgical site infections from a population-based cohort after primary hip and knee arthroplasty in Alberta, Canada. Am J Infect Control 2018;46(10):1123-6. DOI PubMed

18. Ramirez Mendoza JY, Daneman N, Elias MN, Amuah JE, Bush K, Couris CM, Leeb K. A Comparison of Administrative Data Versus Surveillance Data for Hospital-Associated Methicillin-Resistant Staphylococcus aureus Infections in Canadian Hospitals. Infect Control Hosp Epidemiol 2017;38(4):436-43. DOI PubMed

19. Rusk A, Bush K, Brandt M, Smith C, Howatt A, Chow B, Henderson E. Improving Surveillance for Surgical Site Infections Following Total Hip and Knee Arthroplasty Using Diagnosis and Procedure Codes in a Provincial Surveillance Network. Infect Control Hosp Epidemiol 2016;37(6):699-703. DOI PubMed

20. Daneman N, Ma X, Eng-Chong M, Callery S, Guttmann A. Validation of administrative population-based data sets for the detection of cesarean delivery surgical site infection. Infect Control Hosp Epidemiol 2011;32(12):1213-5. DOI PubMed 
21. Leal J, Gregson DB, Ross T, Flemons WW, Church DL, Laupland KB. Development of a novel electronic surveillance system for monitoring of bloodstream infections. Infect Control Hosp Epidemiol 2010 31(7):740-7. DOI PubMed

22. Daneman N, Simor AE, Redelmeier DA. Validation of a modified version of the national nosocomial infections surveillance system risk index for health services research. Infect Control Hosp Epidemiol 2009;30(6):563-9. DOI PubMed

23. Daneman N, Stukel TA, Ma X, Vermeulen M, Guttmann A. Reduction in Clostridium difficile infection rates after mandatory hospital public reporting: findings from a longitudinal cohort study in Canada. PLoS Med 2012;9(7):e1001268. DOI PubMed

24. Lethbridge LN, Richardson CG, Dunbar MJ. Measuring Surgical Site Infection From Linked Administrative Data Following Hip and Knee Replacement. J Arthroplasty 2020;35(2):528-33. DOI PubMed

25. Almond J, Leal J, Bush K, Rogers E, Henderson EA, Ellison J. Hospital-acquired Clostridioides difficile infections in Alberta: the validity of laboratory-identified event surveillance versus clinical infection surveillance. Am J Infect Control 2020;48(6):633-7. DOI PubMed

26. van Mourik MS, van Duijn PJ, Moons KG, Bonten MJ, Lee GM. Accuracy of administrative data for surveillance of healthcare-associated infections: a systematic review. BMJ Open 2015;5(8):e008424. DOI PubMed

27. Freeman R, Moore LS, García Álvarez L, Charlett A, Holmes A. Advances in electronic surveillance for healthcare-associated infections in the 21st Century: a systematic review. J Hosp Infect 2013;84(2):106-19. DOl PubMed

28. Streefkerk HR, Verkooijen RP, Bramer WM, Verbrugh HA. Electronically assisted surveillance systems of healthcare-associated infections: a systematic review. Euro Surveill 2020;25(2):1900321. DOI PubMed

29. Leal J, Laupland KB. Validity of electronic surveillance systems: a systematic review. J Hosp Infect 2008;69(3):220-9. DOI PubMed

30. Donovan TL, Forrester L, Chen Collet J, Wong L, Mori J, Lloyd-Smith E, Ranns B, Han G. Challenging the assertion of comparability of surveillance and administrative data. Infect Control Hosp Epidemiol 2018;39(11):1391-2. DOI PubMed

31. Woeltje KF. Moving into the future: electronic surveillance for healthcare-associated infections. J Hosp Infect 2013;84(2):103-5. DOI PubMed

32. Wilson J, Wloch C, Saei A, McDougall C, Harrington P, Charlett A, Lamagni T, Elgohari S, Sheridan E. Inter-hospital comparison of rates of surgical site infection following caesarean section delivery: evaluation of a multicentre surveillance study. J Hosp Infect 2013;84(1):44-51. DOI PubMed
33. Løwer HL, Eriksen HM, Aavitsland P, Skjeldestad FE. Methodology of the Norwegian Surveillance System for Healthcare-Associated Infections: the value of a mandatory system, automated data collection, and active postdischarge surveillance. Am J Infect Control 2013;41(7):591-6. DOI PubMed

34. Sands K, Vineyard G, Platt R. Surgical site infections occurring after hospital discharge. J Infect Dis 1996;173(4):963-70. DOI PubMed

35. Public Health Ontario. Ontario Agency for Health Protection and Promotion. Provincial Infectious Diseases Advisory Committee. Best practices for surveillance of health care-associated infections In patient and resident populations, 3rd edition. Toronto (ON): Queen's Printer for Ontario; 2014. https://www.publichealthontario.ca/-/media/ documents/B/2014/bp-hai-surveillance.pdf

36. Institut canadien d'information sur la santé. Bases de données clinico-administratives. Évaluation des incidences sur la vie privée, Août 2019. https://www.cihi.ca/sites/default/files/ document/cad-pia-2019-fr-web.pdf

37. Jhung MA, Banerjee SN. Administrative coding data and health care-associated infections. Clin Infect Dis 2009;49(6):949-55. DOI PubMed

38. Drees M, Gerber JS, Morgan DJ, Lee GM. Research Methods in Healthcare Epidemiology and Antimicrobial Stewardship: Use of Administrative and Surveillance Databases. Infect Control Hosp Epidemiol 2016;37(11):1278-87. DOI PubMed

39. Tang KL, Lucyk K, Quan H. Coder perspectives on physician-related barriers to producing high-quality administrative data: A qualitative study. CMAJ Open 5, E617. https://www.cmajopen.ca/content/5/3/E617

40. Du M, Xing Y, Suo J, Liu B, Jia N, Huo R, Chen C, Liu Y. Real-time automatic hospital-wide surveillance of nosocomial infections and outbreaks in a large Chinese tertiary hospital. BMC Med Inform Decis Mak 2014;14(1):9. DOI PubMed

41. Alberta Health Services. Surveillance \& Reporting. Infection Prevention \& Control. https://www.albertahealthservices.ca/ info/Page15736.aspx

42. Institut National de Santé Publique du Québec (INSPQ). Les infections nosocomiales. QC : INSPQ; 2021. https://www. inspq.qc.ca/infections-nosocomiales

43. Gilca R, Hubert B, Fortin E, Gaulin C, Dionne M. Epidemiological patterns and hospital characteristics associated with increased incidence of Clostridium difficile infection in Quebec, Canada, 1998-2006. Infect Control Hosp Epidemiol 2010;31(9):939-47. DOI PubMed

44. Doebbeling BN, Flanagan ME, Nall G, Hoke S, Rosenman M, Kho A. Multihospital infection prevention collaborative: informatics challenges and strategies to prevent MRSA. AMIA Annu Symp Proc 2013;2013:317-25. PubMed 